\title{
LA INVESTIGACIÓN CUALITATIVA (SÍNTESIS CONCEPTUAL)
}

\author{
Miguel Martinez M.*
}

\section{RESUMEN}

El Documento nos brinda un panorama amplio de la investigación cualitativa. Inicia con una revisión necesaria del tema del conocimiento, abordado desde su naturaleza epistemológica para poder entender la totalidad concreta de la realidad, y en la terminología del autor, como un todo polisistémico y la interdisciplinariedad.

En un segundo plano identifica la dimensión dinámica de la investigación cualitativa, en cuanto trata de identificar la naturaleza profunda de las realidades, su estructura y relaciones que se establecen, para cumplir las dos tareas básicas de toda investigación: recoger datos y categorizarlos e interpretarlos.

Hace un tratamiento del marco referencial, los objetivos, las hipótesis y las variables, identificando varios métodos cualitativos, así como los instrumentos y procedimientos.

Palabras claves: Epistemología, Investigación, Hipótesis, Métodos cualitativos, Teorización.

\begin{abstract}
The Document offers an ample panorama us of the qualitative investigation. It initiates with necessary revision of the subject of the knowledge, boarded from his epistemologic nature to be able to understand the concrete totality of the reality, and in the terminology of the author, like an all polisistémico and interdisciplinariedad.

In background it identifies the dynamic dimension of the qualitative investigation, as soon as it tries to identify the deep nature of the realities, his structure and relations that settle down, to fulfill the two tasks basic of all investigation: To gather data and to actegorizar them and to interpret them.

It makes a treatment of the referential frame, the objectives, the hypotheses and the variables, identifying several qualitative methods, as well as the instruments and procedures
\end{abstract}

Keywords: Epistemología, Investigation, Hypotheses, Qualitative methods, Teorización.

* Profesor Honorario de la UNMSM, Profesor Titular (Jubilado) de la Universidad Simón Bolívar (Caracas, Venezuela) y Responsable de la Línea de Investigación «Epistemología y Metodología Cualitativa».

E-mail: miguelm@usb.ve / Web: http://prof.usb.ve/miguelm. 
La vida personal, social e institucional, en el mundo actual, se ha vuelto cada vez más compleja en todas sus dimensiones. Esta realidad ha hecho más difíciles los procesos metodológicos para conocerla en profundidad, conocimiento que necesitamos sin alternativa posible para lograr el progreso de la sociedad en que vivimos. De aquí, ha ido naciendo, en los últimos 25 ó 30 años, una gran diversidad de métodos, estrategias, procedimientos, técnicas e instrumentos, sobre todo en las Ciencias Humanas, para abordar y enfrentar esta compleja realidad. Estos procesos metodológicos se conocen hoy, con el nombre general de Metodologías Cualitativas, y han sido divulgados en un alto número de publicaciones, que van desde unos 400 libros hasta más de 3000 publicaciones parciales (capítulos de libros y artículos de revistas). Estas orientaciones metodológicas tratan de ser sensibles a la complejidad de las realidades de la vida moderna y, al mismo tiempo, estar dotadas de procedimientos rigurosos, sistemáticos y críticos, es decir, poseer una alta respetabilidad científica.

\section{FUNDAMENTACIÓN EPISTEMOLÓGICA}

El gran físico Erwin Schrödinger, premio Nobel por su descubrimiento de la ecuación fundamental de la mecánica cuántica (base de la física moderna), considera que la ciencia actual nos ha conducido por un callejón sin salida y que la actitud científica ha de ser reconstruida, que la ciencia ha de rehacerse de nuevo (1967).

El modelo de ciencia que se originó después del Renacimiento sirvió de base para el avance científico y tecnológico de los siglos posteriores. Sin embargo, la explosión de los conocimientos, disciplinas, especialidades y enfoques que se han dado en el siglo XX y la reflexión epistemológica, encuentran ese modelo tradicional de ciencia no sólo insuficiente, sino, sobre todo, inhibidor de lo que podría ser un verdadero progreso, tanto particular como integrado, de las diferentes áreas del saber.

El problema principal que enfrenta actualmente la investigación en las ciencias sociales, y en general las ciencias humanas y su metodología, tiene un fondo esencialmente epistemológico, pues gira en torno al concepto de "conocimiento" y de "ciencia" y la respetabilidad científica de sus productos: El conocimiento de la verdad y de las leyes de la naturaleza. De aquí, la aparición, sobre todo en la segunda parte del siglo XX, de las corrientes postmodernistas, las posestructuralistas, el construccionismo, el desconstruccionismo, la teoría crítica, el análisis del discurso, la desmetaforización del discurso y, en general, los planteamientos que formula la teoría del conocimiento.

Por ello, nuestro objetivo fundamental aquí, será clarificar e ilustrar que el problema reside en el concepto restrictivo de "cientificidad" adoptado, especialmente en las ciencias humanas, que mutila la legitimidad y derecho a existir de una gran riqueza de la dotación más típicamente humana, como los procesos que se asientan en el uso de la libertad y de la creatividad. Esta gran riqueza de dotación exige en el investigador, por un lado, una gran sensibilidad en cuanto al uso de métodos, técnicas, estrategias y procedimientos para poder captarla, y por el otro, un gran rigor, sistematicidad y criticidad, como criterios básicos de la cientificidad requerida por los niveles académicos.

Este espacio lo han ido tratando de ocupar, a lo largo de la segunda parte del siglo XX, las metodologías cualitativas (cada una en su propio campo y con su especificidad) para lograr 
conocimientos defendibles epistemológica y metodológicamente ante la comunidad científica internacional.

En el ámbito de la experiencia total humana, existe una "experiencia de verdad" (Gadamer, 1984), una vivencia con certeza inmediata, como la experiencia de la filosofía, del arte y de la misma historia, que son formas de experiencia en las que se expresa una verdad que no puede ser verificada con los medios de que dispone la metodología científica tradicional. En efecto, esta metodología usa, sobre todo, lo que Eccles (1985) llama el etiquetado verbal, propio del hemisferio izquierdo, mientras que la experiencia total requiere el uso de procesos gestálticos y estereognósicos, propios del hemisferio derecho.

Según la Neurociencia actual, nuestro sistema cognoscitivo y el afectivo no son dos sistemas totalmente separados, sino que forman un solo sistema, la estructura cognitivo-emotiva; por ello, es muy comprensible que se unan lo lógico y lo estético para darnos una vivencia total de la realidad experienciada. Esto, naturalmente, no desmiente el hecho de que predomine una vez uno y otra el otro, como constatamos en la vida y comportamiento cotidiano de las personas.

La fundamentación y posible salida exitosa de este problema nos la señala el mismo Aristóteles (1973) en su obra máxima, La Metafísica, donde nos advierte que "el ser no se da nunca a nadie en su totalidad, sino sólo según ciertos aspectos y categorías" (Metaf., libro IV). En efecto, toda realidad, y más las realidades humanas, son poliédricas (tienen muchas caras) y sólo captamos, en un momento dado, algunas de ellas. El inculto tiene una captación muy pobre; la persona culta, una mucho más diversificada.

El problema radical que nos ocupa aquí, reside en el hecho de que nuestro aparato conceptual clásico -que creemos riguroso, por su objetividad, determinismo, lógica formal y verificaciónresulta corto, insuficiente e inadecuado para simbolizar o modelar realidades que se nos han ido imponiendo, sobre todo a lo largo del siglo XX, ya sea en el mundo subatómico de la física, como en el de las ciencias de la vida y en las ciencias humanas. Para representarlas adecuadamente necesitamos conceptos muy distintos a los actuales y mucho más interrelacionados, capaces de darnos explicaciones globales y unificadas.

Esta nueva sensibilidad se revela también, a su manera, como ya señalamos, en diferentes orientaciones del pensamiento actual, como la teoría crítica, la condición postmoderna, la postestructuralista y la desconstruccionista, o la tendencia a la desmetaforización del discurso, a un uso mayor y más frecuente de la hermenéutica y de la dialéctica, e igualmente en varias orientaciones metodológicas, como las metodologías cualitativas, la etnometodología, el interaccionismo simbólico, la teoría de las representaciones sociales, el pensamiento sociocéntrico, etc., y vendría a significar el estado de la cultura después de las transformaciones que han afectado a las reglas del juego de la ciencia, de la literatura y de las artes que han imperado durante la llamada "modernidad", es decir, durante los tres últimos siglos.

Los autores de estos movimientos difieren en muchos aspectos, pero tienen también muchas cosas en común, como su ruptura con la jerarquía de los conocimientos y de los valores tradicionales, su bajo aprecio por lo que contribuye a la formación de un sentido universal, su desvalorización de lo que constituye un modelo, y su valoración, en cambio, del racionalismo crítico, de las diferentes lógicas, de la "verdad local", de lo fragmentario, y su énfasis en la subjetividad y en la experiencia estética. 
En fin de cuentas, eso es lo que somos también cada uno de nosotros mismos: un "todo físico-químico-biológico-psicológico-social-cultural-espiritual" que funciona maravillosamente y que constituye nuestra vida y nuestro ser. Por esto, el ser humano es la estructura dinámica o sistema integrado más complejo de todo cuanto existe en el universo. Y cualquier área que nosotros cultivemos debiera tener en cuenta y ser respaldada por un paradigma que las integre a todas.

Estamos poco habituados todavía al pensamiento "sistémico-ecológico". El pensar con esta categoría básica, cambia en gran medida nuestra apreciación y conceptualización de la realidad. Nuestra mente no sigue sólo una vía causal, lineal, unidireccional, sino también, sobre todo, un enfoque modular, estructural, dialéctico, gestáltico, interdisciplinario y estereognósico, donde todo afecta e interactúa con todo, donde cada elemento no sólo se define por lo que es o representa en sí mismo, sino, y especialmente, por su red de relaciones con todos los demás.

La naturaleza es un todo polisistémico que se rebela cuando es reducido a sus elementos. Y se rebela precisamente porque, así, reducido pierde las cualidades emergentes del "todo" y la acción de éstas sobre cada una de las partes.

Este "todo polisistémico", que constituye la naturaleza global, nos obliga incluso a dar un paso más en esta dirección. Nos obliga a adoptar una metodología inter y transdisciplinaria para poder captar la riqueza de la interacción entre los diferentes subsistemas que estudian las disciplinas particulares. No se trata simplemente de sumar varias disciplinas, agrupando sus esfuerzos para la solución de un determinado problema, es decir, no se trata de usar una cierta multidisciplinariedad, como se hace frecuentemente.

La inter y transdisciplinariedad exige respetar la interacción entre los objetos de estudio de las diferentes disciplinas y lograr la integración de sus aportes respectivos en un todo coherente y lógico. Esto implica, para cada disciplina, la revisión, reformulación y redefinición de sus propias estructuras lógicas individuales, ya que esas conclusiones particulares ni siquiera serían "verdad" en sentido pleno. Ejemplos de ello los tenemos a diario en todas las disciplinas, pero, quizá, una de las que más nos afecta personalmente es nuestra medicina actual, que siendo básicamente biológica, ignora la etiología no-biológica de muchas enfermedades y su correspondiente terapéutica, igualmente no biológica.

Pero, podríamos, incluso, ir más allá y afirmar que la mente humana, en su actividad normal y cotidiana, sigue las líneas matrices de este nuevo paradigma. En efecto, en toda elección, la mente estudia, analiza, compara, evalúa y pondera los pro y los contra, las ventajas y desventajas de cada opción o alternativa, y su decisión es tanto más sabia cuantos más hayan sido los ángulos y perspectivas bajo los cuales haya sido analizado el problema en cuestión. Por consiguiente, la investigación científica con el nuevo paradigma consistiría, básicamente, en llevar este proceso natural a un mayor nivel de rigurosidad, de sistematicidad y de criticidad. Esto es precisamente lo que tratan de hacer las metodologías que adoptan un enfoque hermenéutico, fenomenológico, etnográfico, etc., es decir, un enfoque cualitativo que es, en su esencia, estructural-sistémico.

El ser humano es superior a los animales, no por la riqueza de su información sensorial, ya que la mayoría de los animales poseen una agudeza visual, auditiva, olfativa, etc. muy superior a la del hombre, sino por su capacidad de relacionar, interpretar y teorizar con esa información. 
Bajo el punto de vista instrumental, existen hoy más de 60 programas de computación para trabajar con "datos" cualitativos. Los más utilizados son el Atlas.ti, el Ethnograph y el Nud*ist; precisamente, el manejo del primero (de la Universidad de Berlín) ha constituido el objeto completo de estudio de varios de nuestros talleres a nivel de Postgrado. El Atlas.ti es precisamente el más indicado para llevar a cabo la tarea básica que enfrentan muchas investigaciones cualitativas, que tratan de integrar, en una red estructural compleja, las realidades poliédricas que nos presentan los procesos psicológicos, los sociales, los antropológicos, los sociopolíticos y otros. Estas tareas se vuelven casi imposibles de abordar con los procesos normales y simples de la reflexión humana corriente; por eso, la ciencia tradicional ha reducido casi siempre su trabajo a la relación de una o pocas variables: Independientes y dependientes. En las realidades humanas cotidianas biopsicosociales entran normalmente en acción docenas de variables en una interacción recíproca y con muy variados tipos de relaciones. El Atlas.ti, con sus técnicas de categorización, estructuración y teorización, y con los operadores booleanos, semánticos y de proximidad, nos permitirá ir mucho más allá de estas grandes limitaciones. Por ello, ilustramos este programa en el Anexo de nuestra última obra (2004a).

Igualmente, hoy, ya se han desarrollado mucho las "matemáticas de la complejidad" (con centenares de variables interactuantes e intervinientes durante los procesos con la cuarta dimensión "tiempo"), que, gracias a los ordenadores de alta velocidad para resolver problemas complejos, no lineales, trabajan con "relaciones", "formas" y "orden" y grafican diagramas y curvas para descubrir los patrones cualitativos sin ecuaciones ni fórmulas, sino con modelos atractores (tendencias) de alta complejidad. Se trata de unas "matemáticas más cualitativas que cuantitativas". Lo sorprendente de esto es que nuestro hemisferio cerebral derecho trabaja en gran parte de la misma forma, incluso, con una velocidad superior. En tiempos pasados, la orientación científica exigía que se cuantificara el objeto de estudio, que se matematizara, aunque no fuera mensurable; hoy es la Matemática la que ha tenido que respetar y adecuarse a la verdadera naturaleza del objeto, para captarlo como es, en su genuina y compleja naturaleza.

\section{EL ENFOQUE CUALITATIVO}

El término "cualitativo", ordinariamente, se usa bajo dos acepciones. Una, como cualidad: "fulano tiene una gran cualidad: es sincero". Y otra, más integral y comprehensiva, como cuando nos referimos al "control de calidad", donde la calidad representa la naturaleza y esencia completa y total de un producto.

Cualidad y Calidad vienen del mismo término latino qualitas, y éste deriva de qualis (cuál, qué). De modo que a la pregunta por la naturaleza o esencia de un ser: ¿qué es?, ¿cómo es?, se da la respuesta señalando o describiendo su conjunto de cualidades o la calidad del mismo. En sentido propio, filosófico, según Aristóteles, "las acepciones de la cualidad pueden reducirse a dos, de las cuales una se aplica con mayor propiedad y rigor; en efecto, en primer lugar, cualidad es la diferencia o característica que distingue una sustancia o esencia de las otras" (Metafísica, Libro 5, Cap. 14: De la cualidad). Y en la Lógica hace ver que la forma sintética de la cualidad no puede reducirse a sus elementos sino que pertenece esencialmente al individuo y es la que hace que éste sea tal o cual (1973, p. 221). 
Igualmente, el Diccionario de la Real Academia define la cualidad como la "manera de ser de una persona o cosa" ( $2^{\text {a }}$ acepción). Y el Diccionario que acompaña a la Enciclopedia Británica dice que la cualidad "es aquello que hace a un ser o cosa tal cual es" ( $1^{\text {a }}$ acepción, entre 11). Ambos diccionarios siguen el concepto aristotélico.

Es esta acepción, en sentido propio, filosófico, la que se usa en el concepto de "metodología cualitativa". No se trata, por consiguiente, del estudio de cualidades separadas o separables; se trata del estudio de un todo integrado que forma o constituye una unidad de análisis y que hace que algo sea lo que es: Una persona, una entidad étnica, social, empresarial, un producto determinado, etc.; aunque también se podría estudiar una cualidad específica, siempre que se tengan en cuenta los nexos y relaciones que tiene con el todo, los cuales contribuyen a darle su significación propia.

De esta manera, la investigación cualitativa trata de identificar la naturaleza profunda de las realidades, su estructura dinámica, aquella que da razón plena de su comportamiento y manifestaciones. De aquí, que lo cualitativo (que es el todo integrado) no se opone a lo cuantitativo (que es sólo un aspecto), sino que lo implica e integra, especialmente donde sea importante.

\section{LO ESENCIAL DE TODA INVESTIGACIÓN}

Toda investigación, de cualquier enfoque que sea (cualitativo o cuantitativo), tiene dos centros básicos de actividad. Partiendo del hecho que el investigador desea alcanzar unos objetivos, que a veces, están orientados hacia la solución de un problema, los dos centros fundamentales de actividad consisten en:

(1) Recoger toda la información necesaria y suficiente para alcanzar esos objetivos, o solucionar ese problema.

(2) Estructurar esa información en un todo coherente y lógico, es decir, ideando una estructura lógica, un modelo o una teoría que integre esa información. Analógicamente, podríamos decir que todo pende o se apoya en dos pilares centrales, como penden o se apoyan todos los componentes de un puente colgante en sus dos pilares.

También, en forma esquemática y simple, toda investigación se parece un poco al proceso investigativo que realiza un comisario policial al encontrarse ante un crimen consumado, él debe:

(1) Recoger toda la información que pudiera ilustrar lo acaecido, entrevistando a testigos, familiares, amigos, vecinos, compañeros de trabajo, etc.

(2) Estructurar esa información, integrándola en un todo coherente y lógico, por medio de una hipótesis plausible que dé sentido al todo: Un crimen pasional, una venganza, un asalto, una bala perdida, etc.

Un aspecto de gran relevancia es el siguiente: estas dos tareas básicas de (1) recoger "datos" y (2) categorizarlos e interpretarlos, no se realizan siempre en tiempos sucesivos, sino que se entrelazan continuamente. Es decir, que nuestra mente no respeta una secuencia temporal de estas actividades. En efecto, el método básico de toda ciencia es la observación de los "datos" o "hechos" y la interpretación de su significado. Pero la observación y la interpretación son inseparables: resulta inconcebible que una se obtenga en total aislamiento 
de la otra. Toda ciencia trata de desarrollar técnicas especiales para efectuar observaciones sistemáticas y garantizar su interpretación. Sin embargo, sí hay una diferencia de grado: Al principio de la investigación, hay un predominio de la recolección de información sobre la categorización e interpretación; después, a medida que se acerca hacia el final, gradualmente, el balance cambia hacia la categorización e interpretación, con poca recolección de información.

\section{CONCEPTOS FUNDAMENTALES}

\section{Marco epistemológico}

El enfoque cualitativo de investigación es, por su propia naturaleza, dialéctico y sistémico. Estos dos presupuestos, epistemológico y ontológico, conviene hacerlos explícitos, en todo proyecto o desarrollo de investigación, por medio de un breve "marco epistemológico", para evitar los frecuentes malentendidos en los evaluadores de los mismos. Este "marco" se apoyaría básicamente en las ideas expuestas en obras anteriores nuestras (1997b, 1999a, 2004a). Una epistemología de fondo es absolutamente necesaria, ya que es la que le da sentido a la metodología y a las técnicas que se utilicen, como, igualmente, a las reglas de interpretación que se usen.

La teoría del conocimiento o filosofía de la ciencia en que se apoya la metodología cualitativa, rechaza el "modelo especular" (positivista), que considera al sujeto conocedor como un espejo y esencialmente pasivo, al estilo de una cámara fotográfica. Acepta, en cambio, el "modelo dialéctico", considerando que el conocimiento es el resultado de una dialéctica entre el sujeto (sus intereses, valores, creencias, etc.) y el objeto de estudio. No existirían, por consiguiente, conocimientos estrictamente "objetivos". El objeto, a su vez, especialmente en el área de las ciencias humanas, es visto y evaluado (opción o supuesto ontológico) por el alto nivel de complejidad estructural o sistémica, producida por el conjunto de variables bio-psicosociales que lo constituyen. En general, se considera que toda realidad, desde el átomo hasta la galaxia (von Bertalanffy, 1981), está configurada por sistemas de muy alto nivel de complejidad, donde cada parte interactúa con todas las demás y con "el todo" (p. 47).

Estas dos ideas conceptualizadoras (lo dialéctico y lo sistémico) cambiarán la mayoría de los conceptos metodológicos que se apliquen. En efecto, la mayoría de los evaluadores de proyectos o investigaciones cualitativos, suelen hacerlo desde el marco epistemológico del "modelo especular" (científico-positivista), razón por la cual la evaluación falla por la base.

\section{El marco teórico-referencial}

En una investigación cualitativa, este "marco" (así, entre comillas) no debe "enmarcar" (delimitar) la búsqueda del investigador, pues sólo es "referencial", es decir, sólo tiene por finalidad exponer lo que se ha hecho hasta el momento para esclarecer el fenómeno objeto de la investigación. Debe referir las principales investigaciones sobre el área o áreas cercanas: Autores, enfoques y métodos empleados, conclusiones e interpretaciones teóricas a que llegaron, y otros elementos de importancia. En las ciencias humanas, es necesario dar mayor énfasis a lo más cercano (lo regional, lo nacional, lo iberoamericano), ya que 
comparte más nuestra cultura e idiosincrasia. Lo extranjero, especialmente si es anglosajón, podría distorsionar la comprensión de nuestra realidad. En cualquier caso, este "marco" es sólo "teórico-referencial", es decir, fuente de información y nunca modelo teórico en el cual ubicar nuestra investigación. Servirá para contrastar, después (en la etapa de contrastación), nuestras conclusiones con las de esos autores, y así, entenderlas mejor, pero nunca para forzar e imponer una interpretación. Lamentablemente, todo esto es poco entendido en muchos medios académicos, incluso por algunos "metodólogos".

La razón de este proceder es que la utilización de un marco teórico definido nos impone, ya desde el principio, todo un mundo teórico, conceptual e interpretativo que pudiera no ser el más adecuado para entender la realidad que estamos estudiando, sino más bien, un filtro epistemológico que restringe el conjunto de interpretaciones posibles. Y su falta de lógica está en el hecho de que da en gran parte por resuelto lo que todavía no se ha estudiado.

De todos modos, esto no indica que no se pueda hacer una investigación partiendo ya de una teoría sólida (por ej., el psicoanálisis, el marxismo o cualquier otra) y aceptándola, inicialmente, como auténtico marco teórico. El inconveniente de esto está en que, al aceptar ese mundo teórico, conceptual e interpretativo, que pudiera ser incluso, muy ajeno a nuestra realidad, nos estamos jugando el todo por el todo.

Piaget (1976) define este saber "local" que buscamos como "pensamiento sociocéntrico", por oposición al pensamiento técnico y científico: "un saber elaborado para servir a las necesidades, los valores y los intereses del grupo". En este sentido, coincide con el "conocimiento emancipatorio" de Habermas (1982), objeto de la "investigación-acción", y que se opone al "conocimiento instrumental", que es básicamente controlador y explotador.

Moscovici (1984), por su parte, acentúa todavía más esta postura fenomenológica, dándole el rango epistemológico de ciencia (frente y en oposición al conocimiento científico clásico), al considerar las "representaciones sociales" como una forma de conocimiento social específico, natural, de sentido común y práctico, que se constituye a partir de nuestras experiencias, saberes, modelos de pensamiento e información, que recibimos y transmitimos por la tradición, la educación y la comunicación social.

Por consiguiente, es altamente criticable e inaceptable la tendencia antifenomenológica que tienen algunos evaluadores de proyectos e investigaciones, incluso cualitativas, a forzar a los autores de los mismos para que "encuadren" o "enmarquen" sus proyectos o investigaciones en teorías ajenas o en modelos preconcebidos, o para que "definan" los procesos a estudiar con conceptos preestablecidos, traídos de otros contextos y tiempos. Si todo esto se pudiera hacer y respaldar lógicamente, no sería necesario realizar la investigación.

Toda investigación, de cualquier naturaleza que sea (cuantitativa o cualitativa), está constituida por dos etapas: "la recolección de la información necesaria y suficiente" y "la estructuración de esa información". La primera parte de una investigación cualitativa, propiamente dicha, está guiada por varios conceptos, cuya clarificación resumimos a continuación:

\section{Los objetivos}

En las investigaciones cualitativas se fijan unos objetivos a lograr: Algunos son más bien generales y otros específicos, pero todos deben ser relevantes para las personas interesadas en la investigación. A veces, es preferible fijar sólo objetivos generales y determinar los 
específicos durante la marcha, para no buscar metas que quizá resulten triviales o imposibles. El objetivo puede ser muy preciso, como por ejemplo, clarificar tal o cual fenómeno o área problemática, aun cuando sus problemas específicos o dificultades propias estén todavía muy enredados y sólo se puedan plantear o formular expresamente cuando la investigación esté más adelantada.

Estos objetivos determinarán, en parte, las estrategias y procedimientos metodológicos. No obstante, tampoco los objetivos serán intocables. También aquí, al buscar objetivos relevantes, se sigue el famoso principio de "Los tres príncipes de Serendip": "si estás buscando una cosa buena y encuentras otra mejor, deja la primera por la segunda".

La orientación metodológica cualitativa no suele partir del planteamiento de un problema específico, sino de un área problemática más amplia en la cual puede haber muchos problemas entrelazados que no se vislumbrarán hasta que no haya sido suficientemente avanzada la investigación. Por esto, en general, el partir de un problema cierra el posible horizonte que tienen las realidades complejas, como son las realidades humanas.

Esto, de ninguna manera quiere decir que, en un caso específico, no sea útil o conveniente partir de un problema concreto, si eso es particularmente lo que se desea investigar.

\section{La generalización}

Para llegar a la identificación de una estructura humana (psíquica o social) más o menos generalizable, debemos localizar primero esa estructura en individuos o situaciones particulares mediante el estudio y la captación de lo que es esencial o universal, lo cual es signo de lo necesario. Lo universal no es aquello que se repite muchas veces, sino lo que pertenece al ser en que se halla por esencia y necesariamente. La captación de esa esencia depende más de la agudeza intelectual que del uso de técnicas.

Tanto Aristóteles como el mismo Bacon entendían por inducción, no tanto la inferencia de leyes universales a partir de la observación de muchos casos particulares, sino un método mediante el cual llegamos a un punto en el que podemos intuir o percibir la esencia, la forma, o la verdadera naturaleza de las cosas, que encierra lo universal. El mismo Galileo consideraba que las leyes de la naturaleza, que son regulares y que tratamos de descubrir, pueden ser captadas sin necesidad de multiplicar las observaciones, sino que bastaba una buena observación realizada intensivamente para aprehenderlas, como se hace en las ciencias naturales con un solo experimento. Y Piaget, estudiando a fondo a sus propias hijas, estructuró leyes de validez universal que han sido consideradas entre los aportes más significativos de la psicología del siglo XX.

Por otra parte, es necesario tener muy en cuenta que una estructura individual o universal nunca podrá ser inducida del estudio de elementos aislados en muchas personas, del mismo modo que no podemos conocer la fisonomía típica de una determinada raza humana, estudiando de manera separada los ojos, la boca, la nariz, etc., sin ver nunca su red de relaciones en conjunto. Por ese camino ni siquiera reconoceríamos a nuestro mejor amigo. Es precisamente esa "red de relaciones", la que hace que un rostro o una raza sea diferentes de los demás. Sería algo similar a lo que acontece con nuestra propia firma, donde los trazos, rasgos o partes cambian casi siempre, pero la estructura, forma o gestalt permanece la misma, y por eso nos identifica. 
Es muy lógico pensar que el grado de transferibilidad de una situación a otra es una función directa de la similitud que haya entre ambos contextos. Por ello, el esfuerzo mayor del investigador debe dirigirse hacia la identificación del patrón estructural que caracteriza a su objeto de estudio. En cambio, no es él quien debe estudiar el grado de similitud de su contexto con otros contextos o situaciones a los cuales puedan transferirse o aplicarse los resultados de su investigación. Ésa es tarea de quien vaya a hacer la transferencia o aplicación.

Ordinariamente, el enfoque cualitativo no tiene pretensiones de alta generalización de sus conclusiones, sino que, más bien, desea ofrecer resultados y sugerencias para instaurar cambios en una institución, en una empresa, en una escuela o en un grupo o comunidad particular; aunque, evidentemente, al comparar varias investigaciones, se irá logrando un nivel más alto de generalizaciones.

\section{Las hipótesis}

Aunque la mente humana difícilmente trabaja con una ausencia total de hipótesis, en metodología cualitativa, tampoco se formula una hipótesis a verificar, ya que se está abierto a todas las hipótesis plausibles y se espera que la mejor emerja del estudio de los datos y se imponga por su fuerza convincente. Es muy difícil que el investigador tenga la mejor hipótesis a la vista: Si fuera así, no haría falta hacer la investigación. Por ello, es necesaria una gran apertura hacia todas las hipótesis que se vayan revelando consistentes. Las hipótesis son, más bien, provisionales, y se van modificando durante el proceso, para no estrechar nuestra perspectiva y visión de la realidad. En general, no estamos tan interesados en verificar una hipótesis específica, en cuanto que la mejor se revele claramente. Pero también aquí puede ser que un investigador esté interesado en "verificar" una determinada hipótesis, en cuyo caso es lógico que parta de ella.

\section{Las variables y la unidad de análisis}

¿Cuál sería entonces la unidad de análisis, es decir, el objeto específico de estudio de una investigación cualitativa? Sería la nueva realidad que emerge de la interacción de las partes constituyentes, sería la búsqueda de esa estructura con su función y significado. Esta realidad no está en los elementos sino que aparece por las relaciones que se dan entre los elementos, así como surgen las propiedades del agua que no se dan ni en el oxígeno ni en el hidrógeno por separado, o las propiedades del significado al relacionar varias palabras en una estructura lingüística, o la vida por la interacción de varias entidades fisicoquímicas, etc.

No sería, por consiguiente, nada lógico estudiar las variables aisladamente, definiéndolas primero y tratando, luego, de encontrarlas. Es necesario comprender primero o, al menos, al mismo tiempo, el sistema de relaciones en el cual las variables o propiedades se encuentran insertas, enclavadas o encajadas y del cual reciben su propio sentido. También se consideraría improcedente definir las variables operacionalmente, ya que los actos de las personas, en sí, descontextualizados, no tendrían significado alguno o podrían tener muchos significados. El significado preciso lo tienen las "acciones humanas", las cuales requieren para su interpretación, ir más allá de los actos físicos, ubicándolas en sus contextos específicos. El acto en sí no es algo humano; lo que lo hace humano es la intención que lo anima, el significado que tiene para el actor, el propósito que alberga, la meta que persigue; en una 
palabra, la función que desempeña en la estructura de su personalidad y en el grupo humano en que vive.

\section{Las categorías}

El enfoque cualitativo se apoya en la convicción de que las tradiciones, roles, valores y normas del ambiente en que se vive se van internalizando poco a poco y generan regularidades que pueden explicar la conducta individual y grupal en forma adecuada. En efecto, los miembros de un grupo étnico, cultural o situacional comparten una estructura lógica o de razonamiento que, por lo general, no es explícita, pero que se manifiesta en diferentes aspectos de su vida.

No hay, por lo tanto, categorías previas a la investigación, (ni variables, o dimensiones, o indicadores) preconcebidos, ya sea que se consideren independientes o dependientes. Si el investigador las tiene en su mente, es porque las ha tomado de otras investigaciones, de otras muestras realizadas por otros investigadores en otros lugares y en otros tiempos, ya que no existen categorías trascendentes. Las verdaderas categorías que conceptualizarán nuestra realidad deben emerger del estudio de la información que se recoja, al realizar el proceso de "categorización" y durante los procesos de "contrastación" y de "teorización", es decir, cuando se analicen, relacionen, comparen y contrasten las categorías. No obstante, se podría partir de un grupo de categorías preestablecidas, con tal de que se utilicen con mucha cautela y como algo provisional hasta que no se confirmen, y no se deje uno llevar por la tendencia (cosa muy fácil y natural) de rotular la nueva realidad con viejos nombres.

\section{CRITERIOS GENERALES PARA LA ACCIÓN}

Como dice el Premio Nobel de Física, P. Bridgman, "no existe un método científico como tal (...); el rasgo distintivo más fértil de proceder del científico ha sido el utilizar su mente de la mejor forma posible y sin freno alguno" (en: Patton, 1990, p. 140).

El trabajo de campo de la investigación cualitativa camina guiado por algunos criterios que conviene poner adecuadamente de relieve, ya que lo distinguen notablemente de otras clases de investigación.

1. El primero se refiere al lugar donde el investigador debe ir a buscar la información o los "datos" que necesita. El criterio básico para este punto es de carácter general, pero, como no siempre resulta evidente, es necesario enfatizarlo claramente: la información hay que buscarla donde está. Como muchas veces esto altera los planes metodológicos preestablecidos, habrá que tomar conciencia de que primero está la fidelidad a la información que a los planes a seguir. En esta línea de trabajo, es fácil comprender que el investigador a menudo, tiene que tomar decisiones en cuanto a dónde ir, qué datos recoger, con quién hablar, etc. Al contrario de lo que ocurre en las investigaciones con diseños estructurados, aquí la información que se acumula y las estructuras emergentes se usan para reorientar el enfoque y la recolección de nueva información; es decir, que las conjeturas iniciales se van convirtiendo en hipótesis firmes; estas hipótesis comienzan, luego, a ser el centro de la búsqueda de nueva información, estrechando el foco de interés y reorientando las hipótesis. De esa forma, se van perfilando posibles estructuras explicativas y conclusiones teóricas. Como, al 
mismo tiempo, se buscan y contrastan sistemáticamente los casos negativos (situaciones y fenómenos que parecen contradecir u oponerse a las hipótesis y conclusiones), las estructuras teóricas emergentes no sólo son generadas en el campo sino que también son "verificadas" (Williamson, 1981). Todo esto no anula la sistematicidad de la investigación; al revés, exige un orden sistemático altamente fiel a la realidad que emerge del proceso de investigación.

2. El segundo criterio advierte que la observación no debe deformar, distorsionar o perturbar la verdadera realidad del fenómeno que estudia. Tampoco debe descontextualizar los datos aislándolos de su contorno natural. Todo esto exige que la información sea recogida en la forma más completa posible (detalles, matices y aspectos peculiares sobre lenguaje, vestidos, costumbres, rutinas, etc.) y que el estudio sea orientado ecológicamente, en el sentido en que Barker (1968) entiende este término. Por esto, los investigadores cualitativos no definen sus variables a priori, ni mucho menos, se limitan a variables preconcebidas, como hacen los investigadores experimentales, sino que adoptan como estilo una cierta ingenuidad que les permita ver cada aspecto del fenómeno como si fuera nuevo y no familiar y, por lo tanto, potencialmente significativo. De hecho, los datos menos comprensibles resultan luego ser los más significativos. Además, el hecho de que el investigador cualitativo no entra al estudio de campo con un problema completamente planteado o formulado, o con un grupo explícito de hipótesis por verificar, lo pone en la situación de no saber qué datos serán en fin de cuentas importantes y cuáles no lo serán.

3. Como tercer criterio se hace énfasis en que es sumamente conveniente que los procedimientos utilizados permitan realizar las observaciones repetidas veces: para ello, habrá que tratar de grabar las entrevistas, filmar las escenas (si es posible), tomar fotografías, hacer anotaciones pormenorizadas de las circunstancias y situaciones, conservar todos los documentos y hacer, incluso, varias copias de los principales, etc.

4. En cuarto lugar, conviene señalar que, aunque la investigación cualitativa usa muchos tipos de información, la que más busca es aquella que mayor relación tenga y más ayude a descubrir las estructuras significativas que dan razón de la conducta de los sujetos en estudio.

5. En quinto lugar, es necesario distinguir o contrastar la modalidad de cómo otros investigadores recogen los datos sobre un tópico particular, es decir, a través de instrumentos especialmente diseñados para sus fines, y cómo lo hace el investigador cualitativo en su propio campo, generalmente sumergiéndose en el medio que quiere comprender, de tal manera que, brevemente, pudiera definirse su trabajo de campo como la tarea de "vivir su propia vida dentro de otra cultura" (Wolcott, 1975, p. 121).

6. Finalmente, conviene señalar otro criterio más de apreciación, que viene a responder a una objeción frecuente: es un hecho que el observador interactúa con el medio observado y, así, afecta la realidad observada disminuyendo su apreciación "objetiva". Pero, ¿qué concepto tenemos sobre lo que constituye la objetividad científica? El investigador cualitativo no tiene miedo de ser parte de la situación que estudia, de que su presencia parezca "contaminar" los datos, ya que considera imposible recoger datos "absolutos" o "neutrales"; pero trata de tenerlo todo en cuenta, de evaluarlo todo, como el físico que tiene en cuenta la temperatura del termómetro que usa. Él 
sabe que es uno de los actores de la escena; pero el modelo científico que sigue no es el de las ciencias naturales clásicas, sino el de la física moderna, que tiene en cuenta la relatividad general de Einstein y el principio de incertidumbre de Heisenberg, en los cuales el efecto disturbador de la observación sobre lo que es observado se integra en la investigación y en la teoría que de ella se genera.

Estos seis criterios sobre el sistema de un adecuado acopio y manejo de los datos cualitativos, asegura un alto nivel de validez y provee también la base para una cierta forma de confiabilidad o replicabilidad de la investigación.

\section{MÉTODOS CUALITATIVOS}

Como la metodología es, por definición, el camino a seguir para alcanzar conocimientos seguros y confiables y, en el caso de que éstos sean demostrables, también ciencia, la elección de una determinada metodología implica la aceptación de un concepto de "conocimiento" y de "ciencia", es decir, una opción epistemológica (teoría del conocimiento) previa; pero esta opción va acompañada, a su vez, por otra opción, la opción ontológica (teoría sobre la naturaleza de la realidad). La metodología cualitativa está muy consciente de estas dos opciones.

El método cualitativo específico, que se vaya a emplear dependerá de la naturaleza de la estructura a estudiar. La metodología cualitativo-sistémica dispone de una serie de métodos, cada uno de los cuales es más sensible y adecuado que otro para la investigación de una determinada realidad. A continuación, ilustramos la idea central que los caracteriza y diferencia. (Ver los detalles de 12 métodos en Martínez, 2004a, aquí agrupados por su idea central).

Métodos hermenéuticos. En sentido amplio, éstos son los métodos que usa, consciente o inconscientemente, todo investigador y en todo momento, ya que la mente humana es, por su propia naturaleza, interpretativa, es decir, hermenéutica: trata de observar algo y buscarle significado. En sentido estricto, se aconseja utilizar las reglas y procedimientos de estos métodos cuando la información recogida (los datos) necesiten una continua hermenéutica, como sería el caso, por ejemplo, del estudio del crimen organizado, de la dinámica del narcotráfico, de los sujetos paranoicos, etc., donde la información que se nos ofrece puede tratar expresamente de desorientar o engañar. Sin embargo, estos métodos tienen un área de aplicación mucho más amplia: son adecuados y aconsejables, siempre que los datos o las partes de un todo se presten a diferentes interpretaciones.

Métodos fenomenológicos. Estos métodos son los más indicados cuando no hay razones para dudar de la bondad y veracidad de la información y el investigador no ha vivido ni le es nada fácil formarse ideas y conceptos adecuados sobre el fenómeno que estudia por estar muy alejado de su propia vida, como, por ejemplo, el mundo axiológico de los drogadictos o de los homosexuales, las vivencias de las personas en situaciones de vida extremas, la ruptura de una relación amorosa cuando no se ha vivido, una experiencia cumbre (Maslow, 1970), etc.

Métodos etnográficos. Son los de mayor preferencia para entrar a conocer un grupo étnico, racial, de ghetto o institucional (tribu, raza, nación, región, cárcel, hospital, empresa, escuela, y hasta un aula escolar, etc.) que forman un todo muy sui géneris y donde los conceptos de las realidades que se estudian adquieren significados especiales: las reglas, 
normas, modos de vida y sanciones son muy propias del grupo como tal. Por esto, esos grupos piden ser vistos y estudiados globalmente, ya que cada cosa se relaciona con todas las demás y adquiere su significado por esa relación. De ahí que la explicación exige también esa visión global.

El método de investigación-acción. Es el único indicado cuando el investigador no sólo quiere conocer una determinada realidad o un problema específico de un grupo, sino que desea también resolverlo. En este caso, los sujetos investigados participan como coinvestigadores en todas las fases del proceso: planteamiento del problema, recolección de la información, interpretación de la misma, planeación y ejecución de la acción concreta para la solución del problema, evaluación posterior sobre lo realizado, etc. El fin principal de estas investigaciones no es algo exógeno a las mismas, sino que está orientado hacia la concientización, desarrollo y emancipación de los grupos estudiados y hacia la solución de sus problemas.

Recolección de la información. Los instrumentos, al igual que los procedimientos y estrategias a utilizar, los dicta el método escogido, aunque, básicamente, se centran alrededor de la observación participativa y la entrevista semiestructurada. Hay que describir los que se vayan a utilizar y justificarlos. Sin embargo, la metodología cualitativa entiende el método y todo el arsenal de medios instrumentales como algo flexible, que se utiliza mientras resulta efectivo, pero que se cambia de acuerdo al dictamen, imprevisto, de la marcha de la investigación y de las circunstancias.

La muestra a estudiar. Cada uno de los métodos cualitativos (que exponemos detalladamente en otras obras nuestras: 1996b, 1998, 2004, y artículos en nuestra página Web) tiene su forma propia de entender la muestra que nos ofrecerá la información necesaria para realizar la investigación. Pero, en general, la opción ontológica asumida por todos ellos (que es estructural-sistémica) nos exige una muestra que no podrá estar constituida por elementos aleatorios descontextualizados (como es, la mayoría de las veces, la información recogida a través de cuestionarios preconcebidos), sino por "un todo" sistémico con vida propia, como es una persona, una institución, una etnia o grupo social, etc. Por ello, se impone la profundidad sobre la extensión y la muestra se reduce en su amplitud numérica, y se explicitan los criterios conceptuales para su escogencia, según su relevancia para los objetivos de la investigación. Sin embargo, conviene escogerla de forma que estén representadas de la mejor manera posible las variables de sexo, edad, nivel socioeconómico, profesión, etc., según el caso, ya que su información puede ser diferente y hasta contrastante.

La elección de la muestra es de primera importancia, no por lo que representa en sí, sino por la filosofía de la ciencia y los supuestos que implica. De su correcta comprensión depende el significado de toda la investigación. La elección de la muestra dependerá de lo que pensamos hacer con ella y de lo que creemos que se puede hacer con ella. Generalmente, la ciencia busca leyes, conclusiones legaliformes o regularidades, es decir, conocimientos que sean universales o que se puedan generalizar a grupos de casos, personas, poblaciones o situaciones.

A todos nos consta que diferentes personas en diversas posiciones refieren como "los hechos" su versión sobre la misma realidad, y que también varían esa misma información cuando hablan con personas diferentes. Más aún, la misma información puede cambiar continuamente cuando se cambia de grupo informante, y varios documentos sobre el mismo tópico pueden llegar a ser contradictorios. 
La credibilidad de la información puede variar mucho: los informantes pueden mentir, omitir datos relevantes o tener una visión distorsionada de las cosas. Será necesario contrastarla, corroborarla o cruzarla con la de otros, recogerla en tiempos diferentes, usar técnicas de triangulación (combinación de diferentes métodos y fuentes de datos), etc.; conviene, por lo tanto, que la muestra de informantes represente en la mejor forma posible los grupos, orientaciones o posiciones de la población estudiada, como estrategia para corregir distorsiones perceptivas y prejuicios y porque toda realidad humana es poliédrica, tiene muchas caras. Como ya recordamos, Aristóteles decía que "el ser no se da nunca a nadie en su totalidad, sino sólo según ciertos aspectos y categorías" (Metafísica, Lib. iv).

Los tipos de muestras son, básicamente, dos: la muestra estadística o probabilista y la muestra intencional o basada en criterios. Conviene señalar que toda muestra, también la estadística, es siempre intencional o se basa en criterios, aunque diferentes.

En la muestra estadística se extrae, de una población o universo bien definidos, un subgrupo, usando como criterio la condición de que todo miembro tenga exactamente la misma probabilidad de ser elegido. Igualmente, se procura que los estratos sociales y socioeconómicos, la raza, el sexo y demás grupos naturales queden proporcionalmente respetados. Sin embargo, de acuerdo al interés del investigador, la muestra puede ser transversal, longitudinal, de cohorte, de panel, etc.

En la investigación cualitativa, la muestra estadística se considera inapropiada en los siguientes casos: cuando no han sido identificadas todavía las características de la población más amplia, cuando los grupos no están bien delimitados, cuando no se busca la generalización como objetivo importante, cuando las características a estudiar están distribuidas en forma desigual entre los grupos, cuando sólo algunas características de la población son relevantes para el problema en estudio, cuando el investigador no tiene acceso a toda la población.

En la muestra intencional se elige una serie de criterios que se consideran necesarios o altamente convenientes para tener una unidad de análisis con las mayores ventajas para los fines que persigue la investigación. Por ello, se suelen eliminar los casos atípicos o muy peculiares y calibrar muy bien la influencia de todo lo que tiene carácter excepcional; sin embargo, se procura que la muestra represente lo mejor posible los subgrupos naturales, como se indicó para la muestra estadística, y que se complementen y equilibren recíprocamente. Es decir, se trata de buscar una muestra que sea comprehensiva y que tenga, a su vez, en cuenta los casos negativos o deviantes, pero haciendo énfasis en los casos más representativos y paradigmáticos y explotando a los informantes clave (personas con conocimientos especiales, estatus y buena capacidad de información). En conclusión, el investigador tratará de imitar al buen fotógrafo, que busca los mejores ángulos para capturar la mayor riqueza de la realidad que tiene delante.

\section{PROCEDIMIENTOS E INSTRUMENTOS A UTILIZAR}

Como ya señalamos, los instrumentos, al igual que los procedimientos y estrategias a utilizar, los dicta el método escogido, aunque, básicamente, se centran alrededor de la observación directa o participativa y la entrevista semi-estructurada y.

En la actualidad, el investigador con metodología cualitativa, para facilitar el proceso de corroboración estructural, cuenta con dos técnicas muy valiosas: la "triangulación" (de 
diferentes fuentes de datos, de diferentes perspectivas teóricas, de diferentes observadores, de diferentes procedimientos metodológicos, etc.) y las grabaciones de audio y de vídeo, que le permitirán observar y analizar los hechos repetidas veces y con la colaboración de diferentes investigadores.

Si nuestros procedimientos metodológicos deben orientarse hacia el descubrimiento de las estructuras personales o grupales, será necesario tener muy presentes las formas en que se revelan o expresan dichas estructuras. Las técnicas más usadas se centran ordinariamente en el lenguaje hablado o escrito; pero el lenguaje sirve tanto para revelar lo que pensamos y sentimos como también para ocultarlo. Existe, sin embargo, un "lenguaje natural" más universal, el lenguaje no verbal, que es un lenguaje de signos expresivos, como el que se manifiesta a través de los ojos, la mímica, la expresión facial, los movimientos, gestos y posiciones del cuerpo y de sus miembros, el acento, el timbre y el tono de la voz, etc., que depende del sistema nervioso autónomo, es involuntario y casi siempre inconsciente, y contribuye de manera muy eficaz a precisar el verdadero sentido de las palabras, reforzándolo o, quizá, desmintiéndolo.

El investigador cualitativo está muy de acuerdo con la famosa afirmación de Protágoras: "El hombre es la medida de todas las cosas". En efecto, el hombre crea, evalúa y arregla los mismos instrumentos que utiliza, y debe juzgar su buen o mal funcionamiento y la credibilidad de sus datos. En el caso de las investigaciones cualitativas, sin despreciar la ayuda que pueden ofrecerle muchos buenos instrumentos, el observador frecuentemente se convierte en su principal instrumento.

\section{La observación participativa}

Ésta es la técnica clásica primaria y más usada por los investigadores cualitativos para adquirir información. Para ello, el investigador vive lo más que puede con las personas o grupos que desea investigar, compartiendo sus usos, costumbres, estilo y modalidades de vida. Para lograr esto, el investigador debe ser aceptado por esas personas, y sólo lo será en la medida en que sea percibido como "una buena persona", franca, honesta, inofensiva y digna de confianza. Al participar en sus actividades corrientes y cotidianas, va tomando notas de campo pormenorizadas en el lugar de los hechos o tan pronto como le sea posible. Estas notas son, después, revisadas periódicamente con el fin de completarlas (en caso de que no lo estén) y, también, para reorientar la observación e investigación.

Ya que la mayoría de los acontecimientos son expresados o definidos con estructuras lingüísticas particulares, es crucial que el investigador se familiarice con las variaciones del lenguaje y del argot o jerga usados por los participantes, sobre todo cuando éstos son jóvenes. Es, además, importante recoger las historias, anécdotas y mitos que constituyen como el trasfondo cultural-ideológico que da sentido y valor a sus cosas, ya que determinan lo que es importante o no importante, cómo las personas se ven unas a otras y cómo evalúan su participación en los grupos y programas.

¿Qué es, concretamente, lo que el investigador debe hacer al compartir y sumergirse en una observación participativa? Podríamos sintetizar su actividad con el siguiente esquema: el investigador cualitativo debe tratar de responder a las preguntas de quién, qué, dónde, cuándo, cómo y por qué alguien hizo algo; es decir, se consideran importantes los detalles. Este conjunto de interrogantes centran su actividad en la ubicación de los datos más significativos, 
que le servirán después para la interpretación adecuada de los hechos o acontecimientos. También es importante que las expresiones más valiosas y típicas sean recogidas literalmente, para citarlas después entre comillas como testimonio de las realidades observadas.

Además de recoger los datos de la vida diaria, especialmente si se estudia un grupo humano, se debe prestar un cuidado esmerado a los eventos especiales, que serán diferentes de acuerdo a la naturaleza del grupo en estudio: una boda, un rito religioso, un juicio, una graduación, un torneo, un campeonato, una fiesta, un funeral, etc. El análisis de estos eventos manifiesta o revela la estructura o patrón sociocultural de un sistema más amplio del cual forma parte, ya que los eventos especiales se pueden considerar como imágenes que reflejan las estructuras de los grupos, cómo continúan existiendo y por qué perpetúan su existencia. También merecen una atención particular los incidentes clave (riñas, peleas, etc.) por su capacidad informativa.

Como las anotaciones de campo nunca pueden ser muy pormenorizadas, sino, más bien, abreviadas y esquemáticas, conviene detallarlas o ampliarlas el mismo día o al día siguiente, de lo contrario perderán su capacidad de información. Un modo práctico de hacerlo con rapidez consiste en grabar en una cinta un amplio comentario, bien pensado, de las anotaciones tomadas. Estas anotaciones concretas y situacionales serán, además, un testimonio real de la honestidad y "objetividad" de la investigación.

\section{La entrevista semiestructurada}

La entrevista, en la investigación cualitativa, es un instrumento técnico que tiene gran sintonía epistemológica con este enfoque y también con su teoría metodológica.

Esta entrevista adopta la forma de un diálogo coloquial o entrevista semiestructurada, complementada, posiblemente, con algunas otras técnicas escogidas entre las señaladas en nuestras obras $(1996,1998,2004 a)$ y de acuerdo con la naturaleza específica y peculiar de la investigación a realizar. Por esto, vamos a justificar e ilustrar esta técnica con mayor detalle.

La gran relevancia, las posibilidades y la significación del diálogo como método de conocimiento de los seres humanos, estriba, sobre todo, en la naturaleza y calidad del proceso en que se apoya. A medida que el encuentro avanza, la estructura de la personalidad del interlocutor va tomando forma en nuestra mente; adquirimos las primeras impresiones con la observación de sus movimientos, sigue la audición de su voz, la comunicación no verbal (que es directa, inmediata, de gran fuerza en la interacción cara a cara y, a menudo, previa a todo control consciente) y toda la amplia gama de contextos verbales por medio de los cuales se pueden aclarar los términos, descubrir las ambigüedades, definir los problemas, orientar hacia una perspectiva, patentizar los presupuestos y las intenciones, evidenciar la irracionalidad de una proposición, ofrecer criterios de juicio o recordar los hechos necesarios. El contexto verbal permite, asimismo, motivar al interlocutor, elevar su nivel de interés y colaboración, reconocer sus logros, prevenir una falsificación, reducir los formalismos, las exageraciones y las distorsiones, estimular su memoria, aminorar la confusión o ayudarle a explorar, reconocer y aceptar sus propias vivencias inconscientes. Y en cada una de estas posibles interacciones también es posible decidir la amplitud o estrechez con que debe plantearse el problema, si una pregunta debe estructurarse en su totalidad o dejarse abierta, y hasta qué punto resulta conveniente insinuar una solución o respuesta (ver los detalles técnicos en Martínez M., 1996, 1998, 1999c [cap.12], 2004a). 
Así entendida, la técnica de la entrevista es, en gran medida, un arte; sin embargo, lógicamente, las actitudes que intervienen en ese arte son, hasta cierto punto, susceptibles de ser enseñadas y aprendidas; como ocurre en muchos otros campos de la actividad humana, sólo se requiere disposición e interés en aprender.

Kvale (1996) señala que el propósito de la entrevista de investigación cualitativa es obtener descripciones del mundo vivido por las personas entrevistadas, con el fin de lograr interpretaciones fidedignas del significado que tienen los fenómenos descritos. Para ello nos ofrece una guía con diferentes rasgos que caracterizan la entrevista. Igualmente, el mismo autor nos ofrece otros criterios que señalan la preparación o calificación que debe tener $\mathrm{o}$ adquirir un buen entrevistador.

Esta etapa de la investigación cualitativa finalizará cuando se haya recogido y descrito un buen conjunto de material protocolar (primario), en entrevistas, observaciones, grabaciones y anotaciones, que se considere suficiente para emprender una sólida categorización o clasificación que, a su vez, pueda nutrir un buen análisis, interpretación y teorización y conducir a resultados valiosos. Esta segunda etapa, con sus diferentes fases, la describimos en forma detallada en nuestra obra Ciencia y Arte (2004a).

\section{ETAPAS DE LA ESTRUCTURACIÓN}

Esta segunda parte tiene por finalidad describir las etapas y procesos que permitirán la emergencia de la posible estructura teórica, "implícita" en el material recopilado en las entrevistas, observaciones de campo, grabaciones, filmaciones, etc. El proceso completo implica la categorización, la estructuración propiamente dicha, la contrastación y la teorización. Al reflexionar y concentrarse en la información, en esa contemplación, irán apareciendo en nuestra mente las categorías o las expresiones que mejor las describen y las propiedades o atributos más adecuados para especificarlos y, poco a poco, también la estructura teórica que los integra en un todo coherente y lógico.

\section{Categorización}

Si la información señalada, que constituye el material primario o protocolar, es lo más completa y detallada posible, la etapa de la categorización o clasificación exige una condición previa: el esfuerzo de "sumergirse" mentalmente, del modo más intenso posible, en la realidad ahí expresada.

Cada nueva revisión del material escrito, audición de los diálogos o visión de las escenas filmadas, nos permitirá captar aspectos o realidades nuevos, detalles, acentos o matices no vistos con anterioridad o no valorados suficientemente y que, ahora, quizá con otro enfoque o contexto, son determinantes y parecen cambiar o enriquecer el significado. En la práctica, en cada revisión del material disponible es útil ir haciendo anotaciones marginales, subrayando los nombres, verbos, adjetivos, adverbios o expresiones más significativos y que tienen mayor poder descriptivo, poniendo símbolos pictográficos, nemónicos o numéricos, elaborando esquemas de interpretación posible, diseñando y rediseñando los conceptos de manera constante.

Ahora se trata de "categorizar" o clasificar las partes en relación con el todo, de asignar categorías o clases significativas, de ir constantemente integrando y reintegrando el todo y 
las partes, a medida que se revisa el material y va emergiendo el significado de cada sector, evento, hecho o dato. Categorizar es clasificar, conceptualizar o codificar mediante un término o expresión breve que sean claros e inequívocos (categoría descriptiva), el contenido o idea central de cada unidad temática; una unidad temática puede estar constituida por uno o varios párrafos o escenas audiovisuales.

\section{Estructuración}

Toda observación de "algo" es "de ese algo" porque preexisten unos factores estructurantes del pensamiento, una realidad mental fundante o constituyente, un trasfondo $u$ horizonte previo en los cuales se inserta y que le dan un sentido. Si ese marco referencial falta, la observación no es tal, el dato no es dato y el hecho no es nada. Son realidades neutras o plenamente ambiguas.

Todo esto hace que el investigador se aproxime a cualquier expresión de la vida humana, no con la famosa tabula rasa de Locke, sino con expectativas y prejuicios sobre lo que pudiera ser el objeto observado. Debido a ello, la interpretación implica una "fusión de horizontes", una interacción dialéctica entre las expectativas del intérprete y el significado de un texto o acto humano. En términos de la psicología de la Gestalt, aunque no siempre, diríamos que la realidad exterior tiende a sugerirnos la figura, mientras que nosotros le ponemos el fondo (contexto, horizonte, marco teórico).

Heidegger sostiene que "ser humano es ser interpretativo"; es decir, que la interpretación, más que un "instrumento" para adquirir conocimientos, es el modo natural de ser de los seres humanos, y todos los intentos cognoscitivos para desarrollar conocimientos no son sino expresiones de la interpretación sucesiva del mundo.

Por todo ello, es fácil comprender que el proceso de estructuración y teorización constituyen como el corazón de la actividad investigativa: ilustran el procedimiento y el producto de la verdadera investigación, es decir, cómo se produce la estructura o síntesis teórica de todo el trabajo y, también, cómo se evalúa.

Una metodología adecuada para descubrir estructuras teóricas no puede consistir en un procedimiento típicamente lineal, sino que sigue básicamente un movimiento en espiral, del todo a las partes y de las partes al todo, aumentando en cada vuelta el nivel de profundidad y de comprensión. La visión del todo da sentido a las partes y la comprensión de éstas mejora la del todo: conociendo el bosque se comprenden mejor los árboles y, captando las particularidades de éstos, se mejora la comprensión del bosque.

El proceso de estructuración nos lleva, generalmente, a usar metáforas y analogías. Aunque las analogías y los modelos han constituido incuestionablemente una fecunda fuente de teorías científicas, conviene señalar también el peligro que representa su mal uso. Hay analogías fructuosas, pero también engañosas; esto sucede cuando no hay homología estructural entre la realidad simbolizante y la simbolizada.

El mejor modo de comenzar la estructuración es seguir el proceso de integración de categorías menores o más específicas en categorías más generales y comprehensivas. En última instancia, la estructura podría considerarse como una "gran categoría", más amplia, más detallada y más compleja, como el tronco del árbol que integra y une todas las ramas. Igualmente, debe considerarse como una ayuda inestimable la elaboración frecuente de diseños gráficos 
(con flechas, tipos de nexos, relaciones, etc.), ya que permiten integrar y relacionar muchas cosas y ayudan a captarlas en forma simultánea.

\section{Contrastación}

Esta etapa de la investigación consistirá en relacionar y contrastar sus resultados con aquellos estudios paralelos o similares que se presentaron en el marco teórico referencial, para ver cómo aparecen desde perspectivas diferentes o sobre marcos teóricos más amplios y explicar mejor lo que el estudio verdaderamente significa.

Aunque el "marco teórico referencial" sólo nos informa de lo que han realizado otras personas, en otros lugares, en otros tiempos y, quizá, también con otros métodos, sin embargo, el comparar y contraponer nuestras conclusiones con las de otros investigadores, igualmente rigurosos, sistemáticos y críticos, no sólo nos permitirá entender mejor las posibles diferencias, sino que hará posible una integración mayor y, por consiguiente, un enriquecimiento del cuerpo de conocimientos del área estudiada, como se verá en el sector siguiente de la teorización.

Debido a esto, habrá que tener muy presente que el proceso de categorización, análisis e interpretación, deberá estar guiado fundamentalmente por conceptos e hipótesis que provengan o emerjan de la información recabada y de su contexto propio, que muy bien pudieran ser únicos, y no de teorías exógenas, las cuales sólo se utilizarán para comparar y contrastar los resultados propios.

Esta comparación y contrastación pudieran llevarnos hacia la reformulación, reestructuración, ampliación o corrección de construcciones teóricas previas, logrando con ello un avance significativo en el área; es decir, que algunas teorizaciones ya existentes cumplirían en gran parte la función de proveer algunas líneas directrices para interpretar los nuevos datos.

De aquí se deriva la importancia que tiene el diálogo con los autores que nos han precedido en nuestra área de estudio, no para seguir ciegamente lo que ellos digan (marco teórico dogmático), sino para corregir, mejorar, ampliar o reformular nuestras conclusiones; es decir, para enfocarlas desde otros puntos de vista y con el uso de otras categorías, lo cual enriquecerá y profundizará nuestra comprensión de lo que estamos estudiando.

\section{Teorización}

Einstein solía decir que "la ciencia consiste en crear teorías". Pero una teoría es un modo nuevo de ver las cosas, y puede haber muchos modos diferentes de verlas.

El proceso de teorización utiliza todos los medios disponibles a su alcance para lograr la síntesis final de un estudio o investigación. Más concretamente, este proceso tratará de integrar en un todo coherente y lógico, los resultados de la investigación en curso, mejorándolo con los aportes de los autores reseñados en el marco teórico referencial después del trabajo de contrastación.

En el campo de las ciencias humanas, la construcción y reconstrucción, la formulación y reformulación de teorías y modelos teóricos o de alguna de sus partes, mediante elementos 
estructurales de otras construcciones teóricas, es el modo más común de operar y de hacer avanzar estas ciencias.

Einstein mismo llegó a afirmar en repetidas ocasiones que su teoría de la relatividad especial no encontró entidades aisladas ni hechos anteriormente desconocidos, ya que todos sus elementos (los conceptos de espacio, tiempo, materia, fuerza, energía, partículas, gravitación, onda, corpúsculo, velocidad y otros) estaban en el ambiente desde hacía cincuenta años; lo que él propuso fue una nueva manera de clasificar y relacionar cosas ya conocidas. Y Leibniz afirmó: "mi sistema toma lo mejor de todas partes".

La mayoría de los investigadores manifiestan dificultades en describir qué es lo que hacen cuando teorizan; pero un análisis cuidadoso de sus actividades mentales hará ver que son similares a las actividades cotidianas de una persona normal: las actividades formales del trabajo teorizador consisten en percibir, comparar, contrastar, añadir, ordenar, establecer nexos y relaciones y especular; es decir, que el proceso cognoscitivo de la teorización consiste en descubrir y manipular categorías y las relaciones entre ellas.

La transición de los datos a la teoría requiere de imaginación creadora. Popper observa que las teorías son el "resultado de una intuición casi poética" (1963, p. 192). Las hipótesis y teorías científicas no se derivan de los hechos observados, sino que se inventan para dar cuenta de ellos; son conjeturas relativas a las conexiones que se pueden establecer entre los fenómenos estudiados y las uniformidades y regularidades que subyacen a éstos. Las "conjeturas felices" de este tipo requieren gran inventiva, especialmente si suponen una desviación radical de los modos ordinarios del pensamiento científico, como ocurrió con las grandes teorías que fundamentan a cada una de las ciencias.

Einstein precisa que "están en un error aquellos teóricos que creen que la teoría se obtiene inductivamente a partir de la experiencia" (Hanson, 1977, p. 229). Al contrario, una teoría es una construcción mental simbólica, verbal o icónica, de naturaleza conjetural o hipotética, que nos obliga a pensar de un modo nuevo al completar, integrar, unificar, sistematizar o interpretar un cuerpo de conocimientos que hasta el momento se consideraban incompletos, imprecisos, inconexos o intuitivos.

La teoría es, por tanto, un modelo ideal, sin contenido observacional directo, que nos ofrece una estructura conceptual inteligible, sistemática y coherente para ordenar los fenómenos; de manera más concreta, suele consistir en un sistema de hipótesis, fórmulas legaliformes y hasta leyes ya establecidas, de modo que su síntesis puede incluir desde lo plenamente conocido hasta lo meramente sospechado.

\section{CONCLUSIÓN}

Knapp (1986) puntualiza muy bien, como resumen, de toda la Metodología Cualitativa, las actitudes con que debe proceder el investigador cualitativo:

1. Un enfoque inicial exploratorio y de apertura mental ante el problema a investigar.

2. Una participación intensa del investigador en el medio social a estudiar.

3. Uso de técnicas múltiples e intensivas de investigación con énfasis en la observación participativa y en la entrevista con informadores clave. 
4. Un esfuerzo explícito para comprender los eventos con el significado que tienen para quienes están en ese medio social.

5. Un marco interpretativo que destaca el papel importante del conjunto de variables en su contexto natural para la determinación de la conducta, y que pone énfasis en la interrelación global y ecológica de la conducta y de los eventos dentro de un sistema funcional.

6. Resultados escritos en los que se interpretan los eventos de acuerdo con los criterios señalados y se describe la situación con riqueza de detalles y tan vívidamente que el lector pueda tener una vivencia profunda de lo que es esa realidad.

7. Para las demás áreas (métodos y técnicas) de la Metodología Cualitativa, remitimos al lector a revisar la bibliografía que sigue y, de una manera particular, nuestra última obra (2004a) que creemos muy completa y actualizada.

\section{REFERENCIAS BIBLIOGRÁFICAS}

1. Allport, G. (1966). La personalidad: su configuración y desarrollo. Barcelona: Herder.

2. Aristóteles (1973). Obras completas. Madrid: Aguilar.

3. Barker, R. G. (1968). Ecological psychology. California: Stanford University Press.

4. Bertalanffy, L. von (1981). «Historia y situación de la teoría general de sistemas», en Bertalanffy, L. von y otros, Tendencias en la teoría general de sistemas. Madrid: Alianza.

5. Bogdan, R. y S. J., Taylor (1978). Introduction to Qualitative Research Methods. Nueva York: Wiley.

6. Bogdan, R. y Biklen S. (1982). Qualitative research for education: an introduction to theory and methods. Boston: Allyn Bacon.

7. Bridgman, P. (1927). The logic of modern physics. Nueva York: Macmillan.

8. Cook, T. y Reichardt, C. (1986). Métodos cualitativos y cuantitativos en investigación evaluativa. Madrid: Morata

9. Davies, J. T. (1973). The Scientific Approah. Londres: Academic Press.

10. Dilthey, W. (1976). "The rise of hermeneutics", 1900. En Connerton, P. (dir), Critical sociology. Nueva York: Penguin.

11. Eccles, J.C. y Popper, K. (1985). El yo y su cerebro. Barcelona: Labor

12. Gadamer, H. G. (1984). Verdad y método: fundamentos de una hermenéutica filosófica. Salamanca: Sígueme.

13. Habermas, J. (1982). Conocimiento e interés. Madrid: Taurus.

14. Hanson, N. R. (1977). Patrones de descubrimiento. Observación y explicacion. Madrid: Alianza Universidad. 
15. Hegel, G. (1966, orig. 1807). Fenomenología del espíritu. México: F.C.E.

16. Husserl, H. (1962). Ideas relativas a una fenomenología pura y una filosofía fenomenológíca. México: F.C.E.

17. Knapp, S. K. (1986). Etnographic contributions to evaluation research: the experimental schools program evaluation and some alternatives, en Cook y Reichardt, 1986.

18. Kvale, S. (1996). Interviews: An introduction to qualitave research interviewing. Thousand Oaks, CA, Sage.

19. LeCompte, M. y Preissle, J. (1993). Ethnography and qualitative design in educational research, $2^{a}$ edic., San Diego, CA.: Academic Press.

20. Martínez, M. (1986). «La capacidad creadora y sus implicaciones para la metodología de la investigación». En: Psicología (Caracas: UCV), vol.XII, núm.1-2, 37-62.

21. --- (1993). "El proceso creador a la luz de la neurociencia", Comportamiento (Caracas: USB), 2, 1, 3-22.

22. --- (1994). "Hacia un nuevo paradigma de la racionalidad", En Anthropos (Venezuela), núm. 28, 55-78.

23. --- (1996). Comportamiento humano: nuevos métodos de investigación, $2^{\mathrm{a}}$ edic. México: Trillas.

24. --- (1997a). El desafío a la racionalidad científica clásica, Universitas 2000, Vol. 21, N. 3-4, pp.187-200.

25. --- (1997b). El paradigma emergente: hacia una nueva teoría de la racionalidad científica. $2^{\text {a }}$ ed. México: Trillas.

26. --- (1998). La investigación cualitativa etnográfica en educación: Manual teóricopráctico. $3^{\mathrm{a}}$ ed. México: Trillas.

27. --- (1999a). La nueva ciencia: su desafío, lógica y método. México: Trillas.

28. --- (1999b). «Criterios para la superación del debate metodológico cuantitativo/ cualitativo». En: Revista Interamericana de Psicología. 1(33), 79-107.

29. --- (1999c). La psicología humanista: Un nuevo paradigma para la psicología. $2^{\text {a }}$ ed. México: Trillas.

30. --- (2001). «Uso del programa computacional Atlas.ti en la estructuración de 'datos' cualitativos». En: ARGOS. 34, 139-156.

31. --- (2004a). Ciencia y arte en la metodología cualitativa. México: Trillas.

32. --- (2004b). Web = http://prof.usb.ve/miguelm; http://miguelmartinezm.atspace.com

33. Moscovici, S. (1983). «The phenomenon of social representations». En: R.M. Farr y S. Moscovici (dirs): Social representations, Cambridge Univ. Press.

34. ---, (dir.) (1984). Psychologie sociale. París: Preses Universitaires de France.

35. Maslow, A. (1970). Motivation and personality. $2^{\text {a }}$ ed. Nueva York: Harper.. 
36. Patton, M.Q. (1990). Qualitative evaluation and research methods. $2^{\mathrm{a}}$ ed., Newbury Park, CA: Sage.

37. Piaget, J. (1976). Pensée égocentrique et pensée sociocentrique. París: Cahier Vilfredo Pareto, XIV.

38. Popper, K. (1963). Conjetures and refutations. Londres: Routledge.

39. Prigogine, I. (1994). ¿El fin de la ciencia? En: Fried Schnitman, D.

40. Schrödinger, E. (1967). What is the life? \& Mind and Mater. Cambridge Univ. Press.

41. Williamson, J. y otros (1981) (eds). The research craft: an introduction to social research methods. Boston: Scott, Foresman, \& Co.

42. Wolcott, H.F. (1975). "Criteria for an ethnographic approach to research in schools". En: Human Organization, 34, 1975, 111-127. 